\title{
Assessment of Miniature Piezoelectric Travelling-Wave Beam and Plate Robots
}

\author{
By Adel Razek ${ }^{*}$
}

\begin{abstract}
Different up-to-date utilizations have found several benefits in condensing the size of autonomous robots. Miniature traveling wave piezoelectric robots have proven to be appropriate for many of these applications. The principles of locomotion embraced in these robots are mainly inspired by natural biological locomotion and could be categorized by their movement through a specific medium. In this article, after having highlighted the amplifying effect of piezoelectric actuators generating the locomotion necessary for robotic requests, we will review the different types of such locomotion. Next, we will discuss the traveling wave piezoelectric resonant robots. Succeeding, we will look at the operation and usages of piezoelectric beam and plate robots. Finally, we will discuss the modeling aspects implicated in these robots and more generally, the modeling of piezoelectric patches stuck on thin structures.
\end{abstract}

Keywords: piezoelectric, miniature, travelling-wave, locomotion, beam and plate robots

\section{Introduction}

Various recent applications have found many advantages in reducing the size of autonomous robots. We can cite, for example, the storage of cells, research objects in narrow areas, the behavior of swarms, surveillance for security, medical applications. Such miniaturization allows the robot to access restricted scenes such as, water pipes (Zhu 2007), in the middle of debris (Casper and Murphy 2003, Zhang et al. 2012), implanting the human body (Dolghi et al. 2011, Razek 2018, Razek 2019). In addition, due to modest power demands, it is likely that a miniature robot will operate from physical energy sources such as light, electric fields, magnetic fields, or vibration (Paradiso and Starner 2005). One can encounter several difficulties related to the design of a miniature robot. The reduced size of the robot amplifies the complication of the robot's power supply, mechanical scheme, sensors and control. To remedy this, we can reduce the number of actuators or the robot's degrees of freedom.

Piezoelectric actuators are potential allowing technology for autonomous micro robots with locomotion aptitudes matching biological practices. Many piezoelectric robots that move on a solid substrate (smooth and flat ground) have been investigated. Many designs and mechanisms can be found in literature (Avirovik et al. 2014, Baisch 2013, Zhou et al. 2013, Zesch et al. 1995, Cimprich et al. 2006, Simu and Johansson 2002, Ishihara et al. 1995, Uchino 2006, Hariri et

*Emeritus Research Director, C.N.R.S. \& Honorary Professor, CentraleSupelec, GeePs, University of Paris-Saclay and Sorbonne University, France. 
al. 2010, Hariri et al. 2018, Hariri et al. 2015a, Hariri et al. 2015b, Bernard et al. $2014 \mathrm{a}, \mathrm{b})$. The sizes of mobile miniature robots are generally of less than one $\mathrm{dm}^{3}$ and a motion range of at least several times the robots body length.

Piezoelectric materials power miniature robots. Multilayer piezoelectric actuators and bending piezoelectric actuators potentially improve the performance of piezoelectric materials and make them among the most commonly used in miniature mobile robots. Piezoelectric actuators are responsible for movement and are characterized by two energy transformations. These are electrical to mechanical conversion and mechanical-to-mechanical conversion. The first transformation reflects the reverse piezoelectric effect that results in little movement of the mobile miniature robot. The second holds a specific locomotion that amplifies the movement of the miniature robot. The involved locomotion principles are mostly inspired from natural locomotion and could be categorized by their displacement through a fluid medium or on a solid substrate (Hariri 2012).

In this paper, after pointing out the amplifying effect of piezoelectric actuators engendering locomotion that is necessary for robotic applications, we will review the different types of such locomotion. Then, we will discuss the functioning of traveling wave piezoelectric resonant robots. Following this, we will consider the operation and applications of piezoelectric beam and plate robots. Finally, we will discuss the modeling aspects involved in these robots.

\section{Locomotion Principles and Miniature Robots}

As mentioned before, the amplifying effect of piezoelectric actuators engenders locomotion is necessary for robotic applications. In this section, we are going to discuss the two categories of locomotion, displacements on solid surfaces and through fluids.

\section{Locomotion on a Solid Substrate}

The forces associated to on-ground motion involve the gravity, the normal reaction, the friction force and the active force that generates the motion. Locomotion on a solid surface of a mobile miniature robot comprises different types. These are wheeled, walking, inchworm, inertial drive, resonant drive and friction drive.

\section{Wheeled Locomotion}

The principle of this locomotion is founded on small actuators operating wheels. These actuators can be DC motors, step motors, or piezoelectric ones. Cases for wheeled locomotion in miniature robots operated by piezoelectric materials could be found in (Uchino 2006, Epson 2010). 


\section{Walking Locomotion}

This locomotion uses legs that are the drive elements to realize a movement like that of a biological entity. In walking mechanisms of mobile miniature robots, the legs are fastened on the robot by pairs in which each leg can maintain alone the robot equilibrium. The legs perhaps thermal, polymer, electrostatic or piezoelectric drives components. In the last case, piezoelectric actuators are generally multilayer benders or monolithic multilayer. Note that walking is principally a quasi-static locomotion. Different piezoelectric miniature robots involving walking locomotion are given in e.g., Rembold and Fatikow (1997), Simu and Johansson (2002) and Snis et al. (2004).

\section{Inchworm Locomotion}

The inchworm principle is based on operating clampers and extensors actuations permitting displacements (Sunyoto et al. 2006). An inchworm mechanism consists of three actuators, two clampers and one extensor. The extensor one is always between the two clampers. The clamper is used to clamp the device into the substrate while the extensor generates the stroke required for the displacement. Examples of inchworm locomotion in piezoelectric miniature robots are given in Fuchiwaki and Aoyama (2002), Koyanagi et al. (2000), Torii et al. (2001), Yan et al. (2005) and Wood (2008).

\section{Inertial Drive}

The inertial drive notion is engendered in asymmetric actuation situations, i.e., in the case of rapid extension (or contraction) and slow contraction (or extension) of the actuator. Consequently, the control signal of the actuator must be a saw tooth one. Piezoelectric actuators hold high bandwidth that permit such functioning and so, most of miniature robots based on inertial drive principle are actuated by piezoelectric actuators. Two sorts of inertial drive principles can be distinguished: the stick-slip one and the impact drive one (Figure 1). A stick-slip situation consists of an inertial mass that is the main body with legs which are the piezoelectric drive elements. These last are fixed in the inertial mass from one side and exercise the contact surface on the other. An impact drive design consists of an inertial mass connected to the main body via a piezoelectric element (Driesen 2008). Examples of piezoelectric miniature robots based on the inertial drive principles are shown in Rembold and Fatikow (1997), Simu and Johansson (2006), Ikuta et al. (1991) and Zesch et al. (1995).

Figure 1. Stick-Slip (left) and Impact Drive (right) Designs

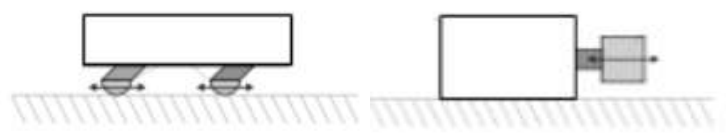

\section{$\underline{\text { Resonant Drive }}$}

Resonant drive motion modes are often used in the case of ultrasonic motors (USM). In the case of mobile miniature robot, the resonant motion is linked to the standing wave type that is defined e.g., in Driesen (2008) by inertial slip 
generation using contact force variation. So, the motion is generated by variation of the contact force, which is the inertial effect of a vertical vibration, which results from the back -and-forth motion of the robot body. This could be abbreviated that the inertial force is engendered by the horizontal vibration of the robot body. Therefore to intensify the inertial force, the horizontal vibration must be increased. This requires increasing the frequency of feet vibration. The motion occurs when this inertial force becomes greater than the maximum feet to substrate friction force. Therefore, it is needed to increase the frequency until a threshold where motion occurs. Examples of piezoelectric mobile miniature robots using this mode could be found in Edqvist et al. (2008), Ferreira and Minotti (1997), Son et al. (2006) and Cimprich et al. (2006).

\section{Friction Drive}

In this case, the origination of motion is due to the change of friction coefficient all through horizontal vibration of the robot body. The variation of friction coefficient results from a no perpendicular contact angle between robot feet and substrate. This is different from the resonant drive. In the case of the resonant drive the horizontal vibration generates the inertial vibration that in turn generates the motion of the robot. While in the friction drive, no inertial force vibration occurs during horizontal vibration but a change in the friction coefficient. This causes a motion in the direction of low friction without involvement of the inertial force. Examples of piezoelectric miniature robots using this mode are given in Aoshima et al. (1993), Ishihara et al. (1995) and Matsuoka et al. (1993).

\section{Locomotion through Fluids}

We consider here locomotion in water and in air (Hariri et al. 2010). The movement in liquid is entirely inspired from natural locomotion and is distributed into locomotion inside liquid and at the liquid surface. For the case inside water, a description of fish swimming modes can be found in Sfakiotakis et al. (1999). Some piezoelectric miniature robots like swimming fishes are developed e.g., in Fukuda et al. (1994), Tzeranis and Papadopoulos (2003), Borgen et al. (2003), Deng and Avadhanula (2005), Kodati et al. (2007), Wiguna et al. (2006) and $\mathrm{Hu}$ et al. (2006), where piezoelectric actuators are used for producing the movements for miniature robots. In addition, piezoelectric actuators are used in Kosa et al. (2007) and Kosa et al. (2008) for creating the travelling wave needed for the movements of swimming microorganisms. Concerning the case of water surface an example of water strider miniature robot walking on water is given in Suhr et al. (2005).

The movement in air for mobile robots is classified into two groups: active air vehicle and passive air vehicle. The first group is divided into three different locomotion principles: flapping, rotary and fixed wing. Different examples for piezoelectric flapping wings are given e.g., in Sitti (2001), Campolo et al. (2003), Park et al. (2006), Nguyen et al. (2007) and Aiguo et al. (2008). 


\section{Traveling Wave Piezoelectric Resonant Robots}

The linear traveling wave ultrasonic motor inspires the idea of these robots (Sashida and Kenjo 1993, Ueha and Tomikawa 1993). This is applied to robotic systems to progress all of the system instead of moving the slider in the case of ultrasonic motors. Various designs were reported in the literature to excite traveling waves in finite structures. Among them we can cite the one mode excitation (see e.g., Kuribayashi et al. 1985) and, the two-mode excitation presented in e.g., Loh and Ro (2000). Both methods are presented for ultrasonic linear motors. Additional methods used to generate traveling waves in finite beam structures, like the feedback control method, active control method and adaptive control method are also present in the literature. Interested readers can refer to Gabai and Bucher (2009), which presents an important review of the excitation of traveling waves in finite beam structures.

Other examples of traveling waves generated on finite beam structures using two Langevin piezoelectric transducers have been investigated. In Hernandez (2010) and Hernandez et al. (2013a) two Langevin piezoelectric transducers are used to create a traveling wave on a finite beam; the traveling wave is created by actuating the two piezoelectric transducers (vibrator-vibrator: two mode excitation) and also by actuating one transducer while the other is used as an absorber (vibrator-absorber: one mode excitation). This was used to realize a prototype of a linear pump system (Hernandez et al. 2010, Hernandez et al. 2013b). Figure 2 illustrates the basis of the mechanical component of this pump that is a linear ultrasonic traveling wave actuator. It is composed of two piezoelectric transducers connected by a metallic flexural beam on which a mechanical traveling wave is induced. Figure 3 shows the fluid-containing component of the pump that is bonded onto the beam. It is composed of three layers of plastic forming two reservoirs connected by a channel whose base is in direct contact with the flexural beam. In Kim et al. (2009) two piezoelectric Langevin transducers were used as vibrators to create a traveling wave on a finite beam. In Loh and Ro (2000) it was demonstrated experimentally the possibility to generate a traveling wave on a finite length using two piezoelectric Langevin transducers as vibrators (two mode excitation). Another type of traveling wave linear ultrasonic motors using piezoelectric patches bonded on an elastic structure as actuators instead of Langevin actuators is also presented in the literature. These types of motors use many piezoelectric patches bonded on one or both sides of the elastic structure and teeth on the structure to generate the traveling wave. As examples of this type of motors, we can cite Bein et al. (1997) and Roh et al. (2001). Dual piezoelectric actuators for the traveling wave ultrasonic motor are presented in Suybangdum et al. (2009). 
Figure 2. Principle of the Mechanical Component of the Pump: $\pi$-Like Structure of a Linear Actuator

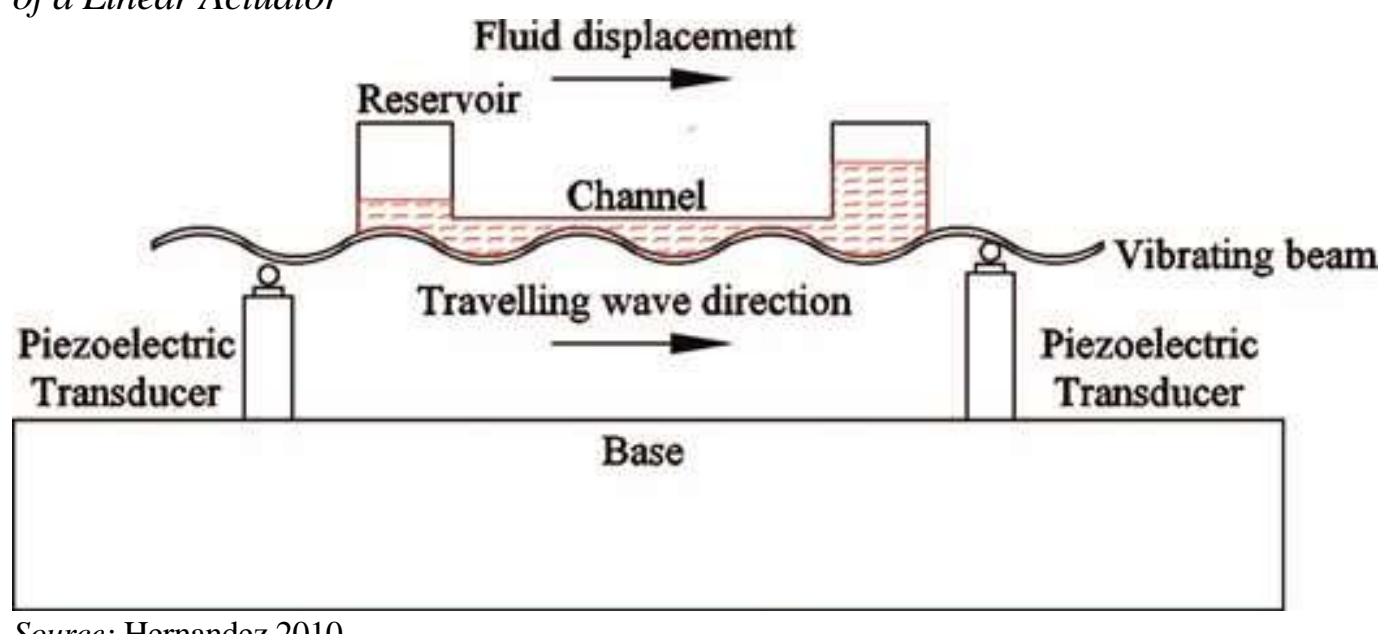

Source: Hernandez 2010.

Figure 3. Fluid-Containing Component of the Pump: (a) Channel Composition and (b) Cross-Sectional View

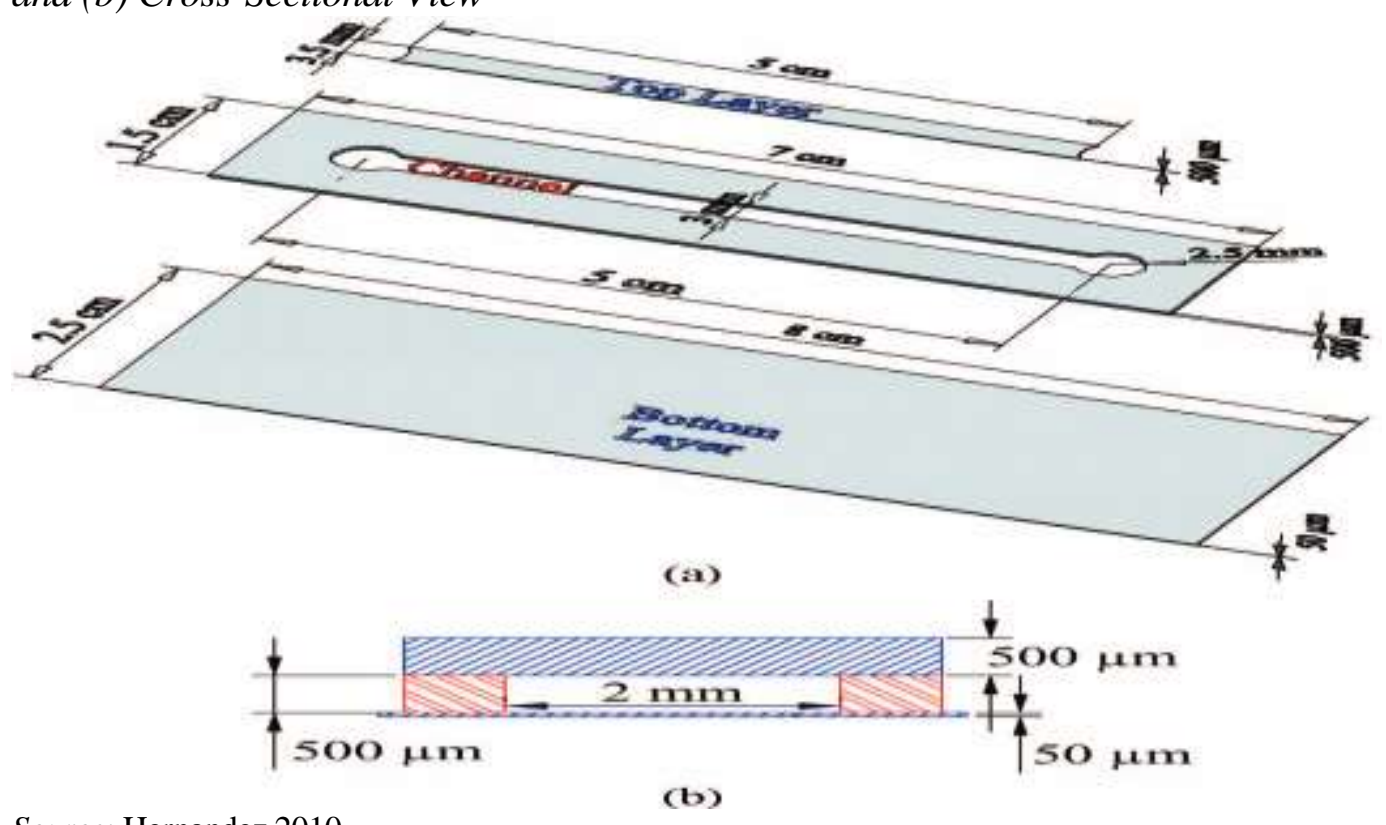

Source: Hernandez 2010.

\section{Thin Structures Involving Piezoelectric Materials}

In general, thin structures containing piezoelectric materials are widely used to control vibrations, for detecting damage in the structure, to design sensors and for energy harvesting. Also to design actuators as: inchworm actuators, micro pumps, valves, miniature robots, motors,..., (Hariri et al. 2010, Hariri et al. 2018, Hernandez et al. 2010, Bernard et al. 2014a, b, Tian et al. 2011, Hariri et al. 2011).

Two large branches are studied in the literature due to their domain of applications, namely beam and plate structures. On the other hand, these systems 
may be symmetrical or asymmetrical where the piezoelectric materials are collocated or not on the thin beam/plate. In a symmetrical system, the piezoelectric materials are bonded face-to-face on both sides of the beam/plate while in an asymmetrical one the piezoelectric materials are bonded only on one side surface of the structure. This non-collocated structure is generally the situation concerned by applications interesting our subject.

\section{Piezoelectric Beam Robots}

In the last sections, we have seen different methods for generating traveling waves in finite beam structures. Concerning applications involving robotics, few works were reported. Nevertheless, in Hariri et al. (2010) and Hariri (2012) a general outline of locomotion principles for piezoelectric miniature robots is detailed. In Son et al. (2006) a robot using standing wave with legs to generate motion, it consists of a piezoelectric layer bonded on a metal layer. Such an organization does not permit the generation of a traveling wave on the beam to move entirely the system. Conversely, in Hariri et al. (2014) a robot consists of only two piezoelectric patches bonded on a beam layer permit, without using teeth or legs, to generate the traveling wave. In such a case, compared to the mentioned robot of Son et al. (2006) and generally the linear ultrasonic motors, the originality lies in the fact that in robotic applications we need to move the entire system and not for e.g., a slider on beam. The motion could be generated using one or twomode excitation. Figure 4 shows the piezoelectric miniature robot of Hariri (2012) and Hariri et al. (2014) for the case of motion generated using two-mode excitation. Figure 5 shows the case of one-mode excitation.

Figure 4. Schematic Diagram of the Piezoelectric Miniature Beam Robot with Two-Mode Excitation
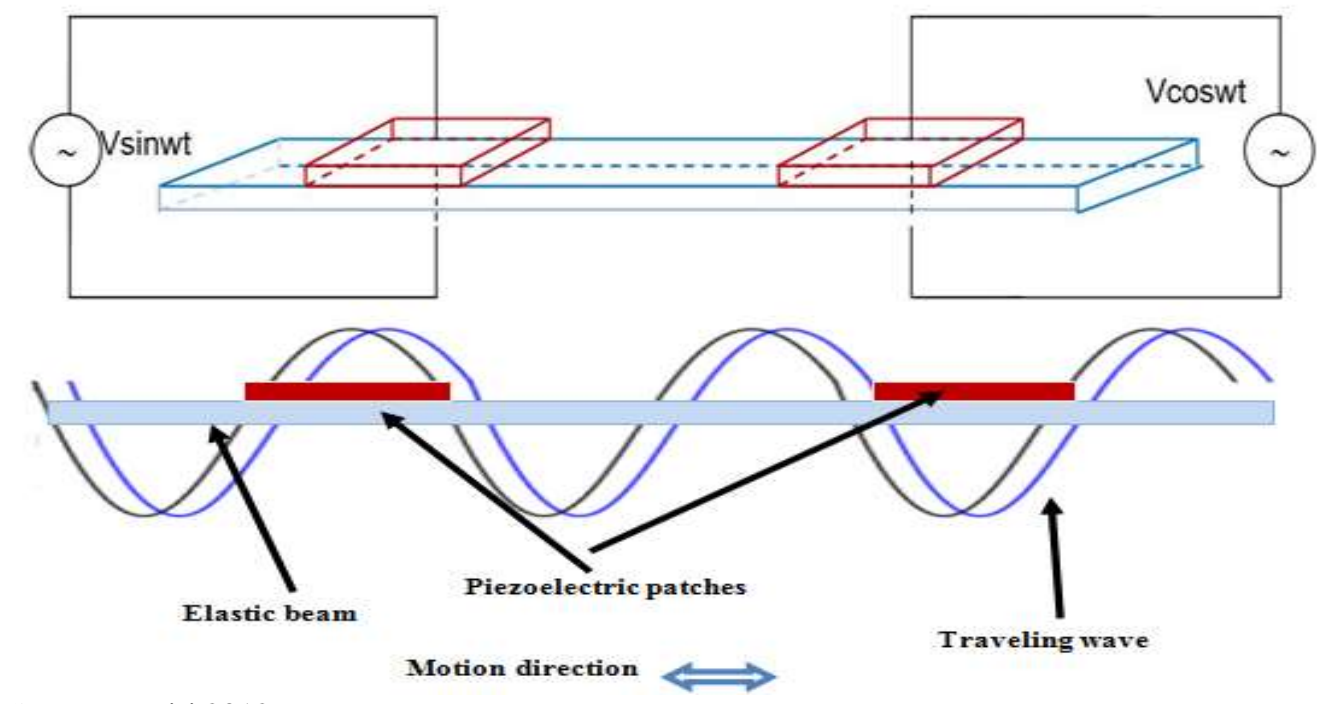

Source: Hariri 2012. 
Figure 5. Beam Robot with One-Mode Excitation

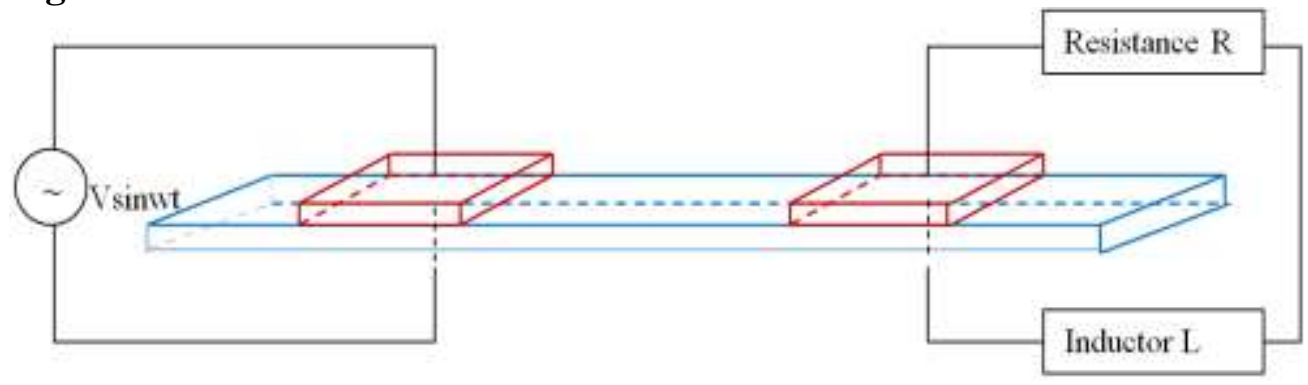

Let us examine theoretically the excitation modes of the miniature piezoelectric beam robot involving two non-collocated piezoelectric patches bonded on the beam surface. In the one-mode excitation, one patch is used as an actuator to engender vibration on the beam while the other is used as a sensor to transform the mechanical vibration into heating and so to generate a traveling wave on the beam. In the two-mode excitation, the two patches are used as actuators to generate the mechanical vibration of the beam in order to create the traveling wave. The details of performing these modes in realistic structures will be illustrated in the next lines.

Pure linear traveling waves are usually observed on long structures. In finite structures similar to beams, the excited vibration wave is partially reflected when it strikes the boundaries. Two approaches can be found in the literature to excite traveling wave in finite structures.

In the first approach one piezoelectric transducer is excited (generally) at the resonance frequency and used to generate the traveling wave on a beam and a second piezoelectric transducer is used to prevent the reflection of the wave by means of a passive electrical circuit. The passive electrical circuit used in this approach can be replaced by active control methods like feedback control or adaptive control to regulate the vibrating wave along the beam (El Moucary et al. 2002). In this case, we have a one-mode excitation at resonance. Therefore, one patch produces the mechanical displacement of the beam by applying an electrical voltage, while the other converts this mechanical displacement into electrical energy, which is then dissipated e.g., through a passive RL electrical network to avoid wave reflection. Note that the inversion of the roles of the two patches leads to inverse the motion direction. The patches positions, the source frequency and the parameters of the dissipative element permit to control the nature of the traveling wave and hence the motion.

The second approach belongs to the active control methods and consists of generating a traveling wave in finite structure. It resides in generating a traveling wave on the structure by one piezoelectric transducer and removes the reflected wave at its boundaries by applying forces using the other piezoelectric transducer. In this two-mode excitation, the two piezoelectric patches produce the mechanical displacement of the beam by applying simultaneously two neighboring natural mode shapes of the beam at the same frequency but with a phase difference of $90^{\circ}$. So, the principle of two-mode excitation is based on the excitation of the two patches at a frequency between two resonance frequencies. At the resonance 
frequency, two progressive waves with the same amplitude propagating in opposite directions cancel each other, resulting in a standing wave on the beam that will stop moving. Below or above the resonance frequency, one progressive wave is excited more than the other is. The resulting waves propagate in the same direction as the waves with the greater amplitude propagate. Note that the motion direction can be reversed by changing phase difference from $90^{\circ}$ to $-90^{\circ}$.

It has been shown in Hariri et al. (2014) and Hariri (2012), that the two-mode excitation shows higher velocity compared to the one- mode case, however the one-mode is better in terms of homogeneity (see Figure 6). This is because that, in the one-mode we excite at the resonance frequency while we excite between two resonance frequencies in the two-mode excitation.

Figure 6. Robot Speed versus Applied Voltage on a Smooth Glass Flat Surface for the One-Mode and Two-Mode Excitation

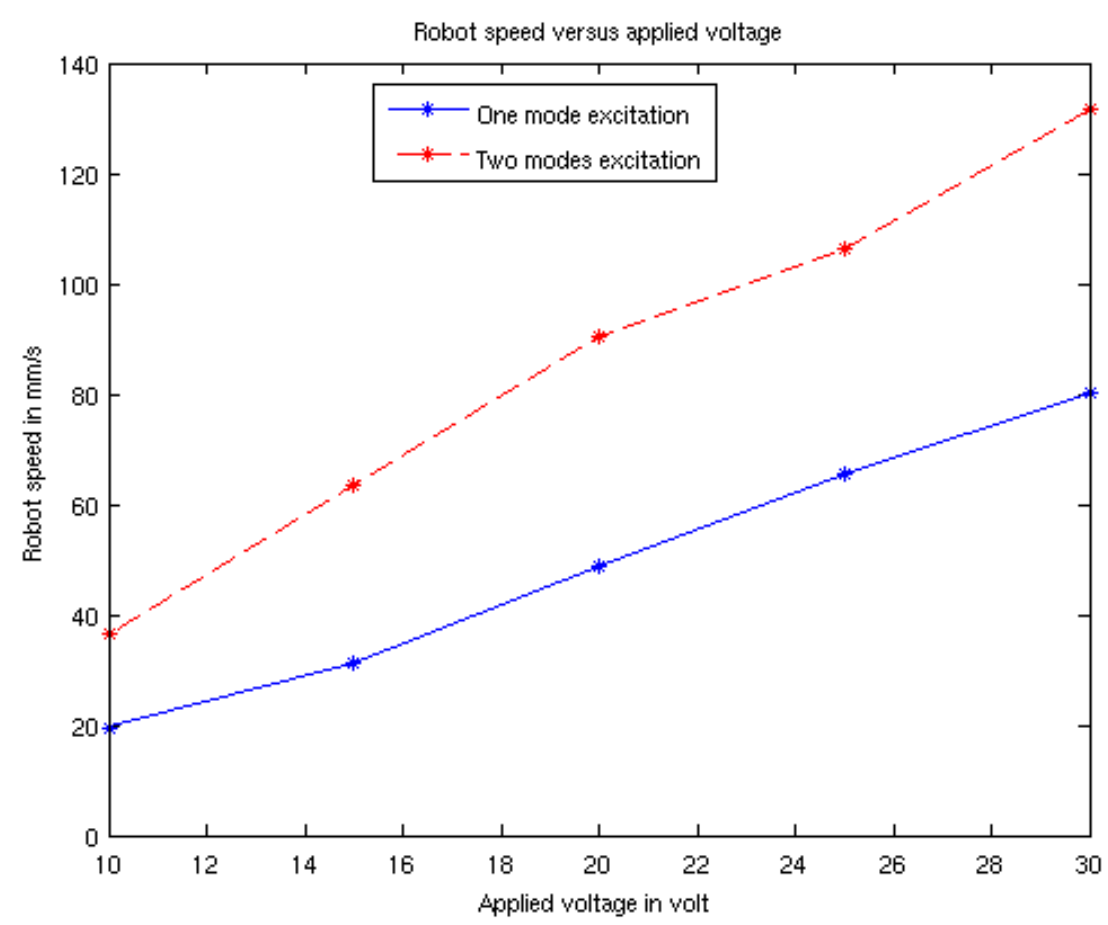

Source: Hariri 2012.

\section{Piezoelectric Patches Bonded on a Plate}

As in the case of beam robots, a miniature piezoelectric plate robot is inspired by linear ultrasonic motors with the difference that there is no slider and the vibrator itself moves like a whole robot and in several directions. Such a robot is within the reach of those who move on a solid substrate (Hariri et al. 2010). We are interested in the mechanism that uses wave propagation to generate movement and control the direction of movement. Two types of waves are used for the propulsion of miniature piezoelectric robots on a solid substrate, the standing wave and the traveling wave. A miniature traveling wave piezoelectric robot is more 
suited to miniaturization than a standing wave robot, the one using legs. For this reason, we will only consider the case of the miniature traveling wave piezoelectric robot.

All of the traveling wave miniature piezoelectric robots mentioned in the last section use a 1D traveling wave on a beam structure for propulsion. However, in this section, a 2D traveling wave on a plate structure is used for propulsion. Such a 2D traveling wave generated on a plate structure is recently reported in Musgrave et al. (2016) for future drag reduction manipulation and to control the interaction between a fluid and the plate structure to improve the efficiency of the systems studied and not to propel the whole system. There is no evidence in this work if this $2 \mathrm{D}$ traveling wave can be used as a driven mechanism to propel the entire system. The objective is to generate a mechanical traveling wave in a finished flexible plate structure to propel the plate in different directions using piezoelectric patches (Hariri et al. 2018). Figure 7 shows non-collocated (stuck on the same side) piezoelectric patches bonded on a plate. The objective in Hariri et al. (2018) is to generate a traveling wave in a plate structure to move it on a solid substrate using piezoelectric patches stuck on the same face of the plate surface.

Figure 7. Non-Collocated Piezoelectric Patches Bonded on a Plate

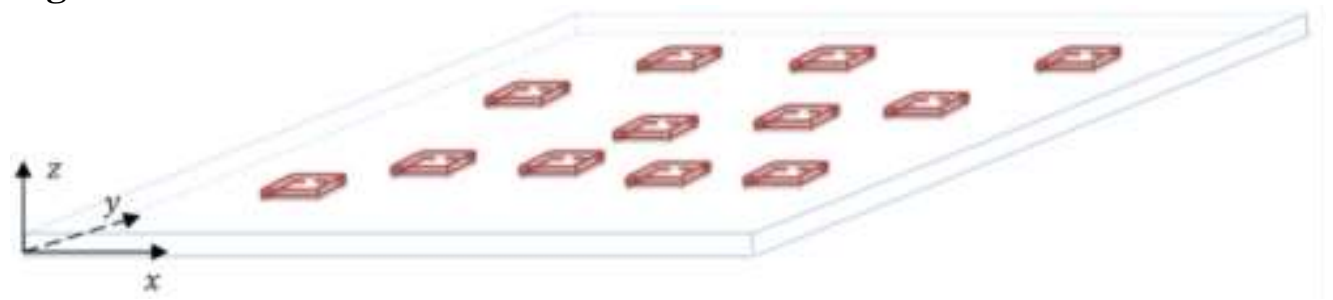

Source: Hariri 2012.

The design proposed in Hariri et al. (2018) consists of an elastic thin plate structure, with four non-collocated piezoelectric patches bonded to its surface. This system is called traveling wave driven piezoelectric plate robot for planar motion. The traveling wave on the plate is generated using the "two-mode excitation" method defined in the last section. The piezoelectric patches are used in this case to excite the plate at a frequency between two resonant frequencies in order to generate a traveling wave on the plate. The traveling wave is at the origin of the frictional forces exerted at the level of the contact lines between the plate and the ground that lead to the movement of the robot in different directions.

The design process in Hariri et al. (2018) consists in determining the optimal geometry and the mechanical properties of the plate robot that lead to optimize the generation of the traveling wave on it. It involves determining the optimal shape and geometry of the piezoelectric plate and patches, the material used for the plate and piezoelectric patches, and the locations of the piezoelectric patches. The type of material used for the plate is studied in Hariri et al. (2015a) where a comparison is made between steel, aluminum, brass and acrylic. It has been found that at a plate thickness of $0.5 \mathrm{~mm}$, aluminum is the best elastic material, which presents the best compromise between maximum transverse displacement and maximum locking force. The effect of the positions of the patches on the performances of the 
traveling wave is studied in Hariri et al. (2015b) in the case of a beam structure. It has been found that locating the patches near the ends of the beam structure would lead to better performance and that the traveling wave is mainly generated over the distance between the patches. The difference in traveling wave performance for different patch positions was due to the establishment of the patches relative to nodes and anti-nodes of a given frequency.

It has been shown in Hariri et al. (2018) and Hariri (2012) that in the case of plates a traveling wave generated on a thin plate using distributed piezoelectric patches can move the plate in multi degree of freedom.

\section{Modeling of Piezoelectric Patches Bonded on Thin Structures}

In the last two sections, we studied the cases of piezoelectric patches bonded on beams and plates. In the case of plates, we discussed the case of an elastic thin plate structure, with non-collocated piezoelectric patches bonded to its surface. The design and optimization of such structure needs 2D modeling of noncollocated patches bonded on thin structure (Hariri et al. 2015a).

For symmetrical or asymmetrical beam structures with respectively collocated or non-collocated piezoelectric materials, a 1D analytical or numerical model can be used to model such system (see Figure 6). In the case of plate structures, 2D or 3D Finite Element Method (FEM) can be used to model the system (Ouchetto et al. 2007, Ren and Razek 1990, Rapetti et al. 2002, Carpes et al. 2000, Razek 2019). In the 3D approach, volume elements are used while in the 2D case surface elements are used while the third dimension is introduced in the model equations. It is obvious that the second approach is faster but a little more complicated in model formulation. An example illustrating such 2D modeling is given in Hariri (2012) and Hariri et al. (2015a). The studied device consists of a thin structure with several piezoelectric patches bonded on one side of its surface i.e., non-collocated piezoelectric patches (asymmetrical system). Examples of such asymmetric systems finite element meshes are shown in Figure 8 where four colored rectangles stand for piezoelectric patches are bonded on one side of rectangular and circular thin structures with and without holes. It is worthy to note that all modeling tools are supposed to be validated by observation experience (Razek 2020). 
Figure 8. Finite Element Meshes of Non-Collocated Piezoelectric Patches Bonded on Structures

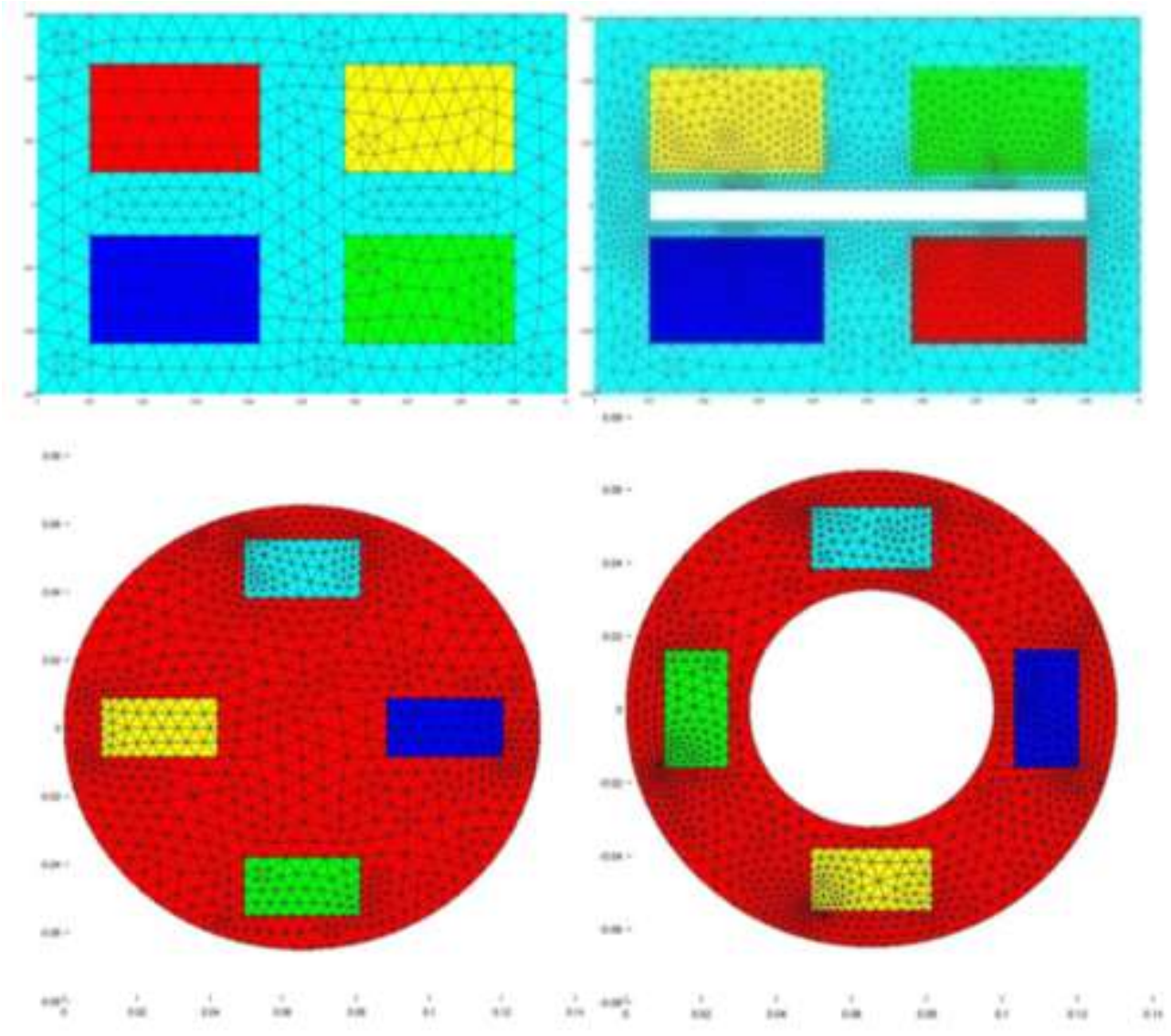

Source: Hariri 2012.

\section{Conclusions}

This paper has reviewed the different types of locomotion perhaps used in robotics. The functioning of traveling wave piezoelectric resonant robots was discussed. The operation and applications of piezoelectric beam and plate robots have been assessed through numerous published works opting for those with innovative characteristics.

These use traveling waves practiced by non-collocated patches bounded on flexible thin beams or plates. These patches are activated by one or two-mode excitations. In the case of beams, the two-mode excitation shows higher velocity compared to the one-mode case, however the one-mode is better in homogeneity. This is because in the one-mode excitation we excite at the resonance frequency while we excite between two resonance frequencies in the two-mode excitation.

In addition, in the case of plates a traveling wave generated on a thin plate using distributed piezoelectric patches can move the plate in multi degrees of freedom.

The modeling aspects involved in these robots and more generally, the 
modeling of piezoelectric patches bonded on thin structures have exposed realistic tools.

\section{References}

Aiguo M, Yawen H, Fukushima Y, Shimojo M (2008) Development of an active flapping wing using piezoelectric fiber composites. In IEEE International Conference on Robotics and Biomimetics ROBIO, 2144-2149.

Aoshima SI, Tsujimura T, Yabuta T (1993) Miniature mobile robot using piezo vibration for mobility in a thin tube. Journal of Dynamic Systems, Measurement and Control, Transactions of the ASME 115(2 A): 270-278.

Avirovik D, Butenhoff B, Priya S (2014) Millipede-inspired locomotion through novel Ushaped piezoelectric motors. Smart Materials and Structures 23(3): 1-5.

Baisch AT (2013) Design, manufacturing and locomotion studies of ambulatory microrobots. PhD Thesis. Cambridge, Massachusetts: Harvard University.

Bein T, Breitbach EJ, Uchino K (1997) A linear ultrasonic motor using the first longitudinal and the fourth bending mode. Smart Materials and Structures 6(1997): 619-627.

Bernard Y, Hernandez C, Razek A (2014a) Radial traveling wave ultrasonic motor design. Actuator 2014(Jun): 663-666.

Bernard Y, Hariri H, Razek A (2014b) Mobile piezoelectric device . Patent, WO Patent $\mathrm{n}^{\circ}$ : EP2014/072867 and WO2015059283 A1.

Borgen MG, Washington GN, Kinzel GL (2003) Design and evolution of a piezoelectrically actuated miniature swimming vehicle. IEEE/ASME Transactions on Mechatronics 8(1): 66-76.

Campolo D, Sahai R, Fearing R (2003) Development of piezoelectric bending actuators with embedded piezoelectric sensors for micromechanical flapping mechanisms. In IEEE International Conference on Robotics and Automation 3: 3339-3346.

Carpes WP, Pichon L, Razek A (2000) A 3D finite element method for the modelling of bounded and unbounded electromagnetic problems in the time domain. International Journal of Numerical Modelling: Electronic Networks, Devices and Fields 13(6): 527-540.

Casper J, Murphy RR (2003) Human-robot interactions during the robot-assisted urban search and rescue response at the World Trade Center. IEEE Transactions on Systems, Man, and Cybernetics. Part B, Cybernetics 33(3): 367-85.

Cimprich T, Kaegi F, Drieseu W, Ferreira A, Breguet J (2006) Ultrasonic monolithic piezoelectric multi DOF actuators for mobile micro robots. In ACTUATOR '06. Bremen, DE.

Deng X, Avadhanula S (2005) Biomimetic micro underwater vehicle with oscillating fin propulsion: system design and force measurement. In Proceedings of the 2005 IEEE International Conference on Robotics and Automation ICRA, 3312-3317.

Dolghi O, Strabala KW, Wortman TD, Goede MR, Farritor SM, Oleynikov D (2011) Miniature in vivo robot for laparoendoscopic single-site surgery. Surgical Endoscopy 25(10): 3453-3458.

Driesen W (2008) Concept, modeling and experimental characterization of the modulated friction inertial drive (MFID), locomotion principle: application to mobile microrobots. $\mathrm{PhD}$ Thesis. Lausanne, Switzerland: Swiss Federal Institute of Technology Lausanne. 
Edqvist E, Snis N, Sjölund M, Murase T, Söderberg A, Johansson S (2008) The assembly of millimeter sized mass producible autonomous robots. In Proceedings of the International Conference on New Actuators, ACTUATOR '08, 304-307.

El Moucary C, Mendes E, Razek A (2002) Decoupled direct control for PWM inverter-fed induction motor drives. IEEE Transactions on Industry Applications 38(5): 13071315.

Epson (2010) News release: Epson to unveil a prototype microrobot with ultra-thin ultrasonic motor and power-saving Bluetooth module. Epson.

Ferreira A, Minotti P (1997) Control of a multidegree of freedom standing wave ultrasonic motor driven precise positioning system. Review of Scientific Instruments 68(4): 1779-1786.

Fuchiwaki O, Aoyama H (2002) Piezo based micro robot for microscope instrument. In Proceedings of the International Conference on Mechatronics Technology, ICMT '02, 499-503.

Fukuda T, Kawamoto A, Arai F, Matsuura H (1994) Mechanism and swimming experiment of micro mobile robot in water. In Proceedings of 1994 IEEE International Conference on Robotics and Automation 1, 814-819.

Gabai R, Bucher I (2009) Excitation and sensing of multiple vibrating traveling waves in one-dimensional structures. Journal of Sound and Vibration 319(1-2): 406-425.

Hariri $\mathrm{H}$ (2012) Design and realization of a piezoelectric mobile for cooperative use. $\mathrm{PhD}$ Thesis. Paris, France: Université Paris Sud-XI (GeePs).

Hariri H, Bernard Y, Razek A (2010) Locomotion principles for piezo- electric miniature robots. In Proceedings of ACITUATOR 10, 1015-1020. Bremen, DE.

Hariri H, Bernard Y, Razek A (2011) Analytical and finite element model for unimorph piezoelectric actuator: actuator design. In Piezo2011, 71-75. Sestriere, Italy.

Hariri H, Bernard Y, Razek A (2014) A traveling wave piezoelectric beam robot. Smart Materials and Structures 23(2): 025013.

Hariri H, Bernard Y, Razek A (2015a) A two dimensions modeling of non-collocated piezoelectric patches bonded on thin structure. Curved and Layered Structures 2(1): $15-27$.

Hariri H, Bernard Y, Razek A (2015b) Dual piezoelectric beam robot: the effect of piezoelectric patches' positions. Journal of Intelligent Material Systems and Structures 26(18): 2577-2590.

Hariri H, Bernard Y, Razek A (2018) 2-D Traveling wave driven piezoelectric plate robot for planar motion. IEEE/ASME Transactions on Mechatronics 23(1): 242-251.

Hernandez C (2010) Realization of piezoelectric micro pumps. PhD Thesis. Paris, France: University Paris sud-XI (GeePs).

Hernandez C, Bernard Y, Razek A (2010) A global assessment of piezoelectric actuated micro-pumps - A review. The European Physical Journal Applied Physics 51(2): 20101.

Hernandez C, Bernard Y, Razek A (2013a) Design and manufacturing of a piezoelectric traveling-wave pumping device. IEEE Transactions on Ultrasonic, Ferroelectrics, and Frequency Control 60(9): 1949-1956.

Hernandez C, Bernard Y, Razek A (2013b) Ultrasonic traveling wave micro pump for liquid. US Patent App. Patent $n^{\circ}$ : US-2013-02423627.

Hu T, Wang G, Shen L, Li F (2006) A novel conceptual fish-like robot inspired by Rhinecanthus Aculeatus. In Ninth International Conference on Control, Automation, Robotics and Vision ICARCV '2006, 1-5.

Ikuta K, Kawahara A, Yamazumi S (1991) Miniature cybernetic actuators using piezoelectric device. In Proceedings IEEE Micro Electro Mechanical Systems, MEMS '91, 131-136. 
Ishihara H, Fokuda T, Kosuge K, Arai F, Hamagishi K (1995) Approach to distributed micro robotic system development of micro line trace robot and autonomous micro robotic system. In IEEE International Conference on Robotics and Automation, ICRA.95 1, 375-380.

Kim GH, Park JW, Jeong SH (2009) Analysis of dynamic characteristics for vibration of flexural beam in ultrasonic transport system. Journal of Mechanical Science and Technology 23(May): 1428-1434.

Kodati P, Hinkle J, Deng X (2007) Micro Autonomous Robotic Ostraciiform (MARCO): design and fabrication. In IEEE International Conference on Robotics and Automation, 960-965.

Kosa G, Shoham M, Zaaroor M (2007) Propulsion method for swimming micro robots. IEEE Transactions on Robotics 23(1): 137-150.

Kosa G, Jakab P, Hata N, Jolesz F, Neubach Z, Shoham M, et al. (2008) Flagellar swimming for medical micro robots: theory, experiments and application. In 2 nd IEEE RAS \& EMBS International Conference on Biomedical Robotics and Biomechatronics BioRob.

Koyanagi T, Fukui R, Torii A, Ueda A (2000) Miniature robots with three degrees of freedom. In Proceedings of the IEEE International Symposium on Micromechatronics and Human Science MHS'00, 207-212.

Kuribayashi M, Ueha S, Mori E (1985) Excitation conditions of a flexural traveling waves for a reversible ultrasonic linear motor. The Journal of the Acoustical Society of America 77(4): 1431-1435.

Loh BG, Ro PI (2000) An object transport system using flexural ultrasonic progressive waves generated by two modes excitation. IEEE Transactions on Ultrasonic, Ferroelectric, and Frequency Control 47(4): 994-999.

Matsuoka T, Asano M, Fukushima H, Okamoto H, Uchida M, Matsui T (1993) Mechanical analysis of micro mobile machine with piezoelectric element. In Proceedings of the IEEE/RSJ International Conference on Intelligent Robots and Systems IROS '93, 1685-1690.

Musgrave PF, Malladi VNS, Tarazaga PA (2016) Generation of traveling waves in a 2D plate for future drag reduction manipulation. In Book on Special Topics in Structural Dynamics, 6, Part of the Series Conference Proceedings of the Society for Experimental Mechanics Series, 129-138.

Nguyen QV, Park HC, Goo NS, Byun D (2007) A flying insect-like flapper actuated by a compressed LIPCA. In IEEE International Conference on Robotics and Biomimetics ROBIO, 19-24.

Ouchetto O, Zouhdi S, Bossavit A, Griso G, Miara B, Razek A (2007) Homogenization of structured electromagnetic materials and metamaterials. Journal of Materials Processing Technology 181(1-3): 225-229.

Paradiso J, Starner T (2005) Energy scavenging for mobile and wireless electronics. IEEE Pervasive Computing 4(1): 1-27.

Park HC, Syaifuddin M, Goo NS, Byun D, Yoon KJ (2006) An insect-mimicking flapping system actuated by a piezo ceramic actuator. In IEEE International Conference on Robotics and Biomimetics, 451-456.

Rapetti F, Maday Y, Bouillault F, Razek A (2002) Eddy-current calculations in threedimensional moving structures. IEEE Transactions on Magnetics 38(2): 613-616.

Razek A (2018) Towards an image-guided restricted drug release in friendly implanted therapeutics - Review. The European Physical Journal of Applied Physics 82(3): 31401.

Razek A (2019) Assessment of supervised drug release in cordial embedded therapeutics. Athens Journal of Technology and Engineering 6(2): 77-91. 
Razek A (2020) The observable, the theory, and prospective revised models for societal concerns. Athens Journal of Sciences 7(1): 1-14.

Rembold U, Fatikow S (1997) Autonomous microrobots. Journal of Intelligent and Robotic Systems 19(4): 375-391.

Ren Z, Razek A (1990) A coupled electromagnetic-mechanical model for thin conductive plate deflection analysis. IEEE Transactions on Magnetics 26(5): 1650-1652.

Roh Y, Lee S, Han W (2001) Design and fabrication of a new traveling wave-type ultrasonic linear motor. Sensors and Actuators A: Physical 94(3): 205-210.

Sashida T, Kenjo T (1993) An Introduction to Ultrasonic Motors. (Oxford: Clarendon).

Sfakiotakis M, Lane DM, Davies JBC (1999) Review of fish swimming modes for aquatic locomotion. IEEE Journal of Oceanic Engineering 24(2): 237-252.

Simu U, Johansson S (2002) Fabrication of monolithic piezoelectric drive units for a miniature robot. Journal of Micromechanics and Microengineering 12(5): 582-589.

Simu U, Johansson S (2006) Analysis of static and dynamic motion mechanisms for piezoelectric miniature robots. Journal of Sensors and Actuators 132(2): 632-642.

Sitti M (2001) PZT actuated four-bar mechanism with two flexible links for micromechanical flying insect thorax. In Proceedings ICRA. IEEE International Conference on Robotics and Automation 4, 3893-3900.

Snis N, Simu U, Johansson S (2004) Piezoelectric drive platform for $\mathrm{cm} 3$ sized autonomous robot. In Proceedings of the International Conference on New Actuators, ACTUATOR '04, 106-109.

Son K, Kartik JV, Wickert JA, Sitti M (2006) An ultrasonic standing-wave-actuated nanopositioning walking robot: piezoelectric-metal composite beam modelling. Journal of Vibration and Control 12(12): 1293-1309.

Suhr SH, Song YS, Lee SJ, Sitti M (2005) Biologically inspired miniature water strider robot. In Proceedings of the Robotics: Science and Systems I, 319-325. Boston, U.S.A.

Sunyoto S, Bernard Y, Razek A (2006) Design and realization of a linear piezoelectric actuator for orthopedic applications. Journal of Advanced Science 18(1/2): 162-165.

Suybangdum P, Smithmaitrie P, Laoratanakul P (2009) Dual piezoelectric actuators for the traveling wave ultrasonic linear motor. In Fourth International Conference on Experimental Mechanics.

Tian Y, Hernandez C, Bernard Y, Razek A (2011) Self moving stick slip piezoelectric actuator design and modeling. In Piezo11. February 2011, Sestriere, Italy.

Torii A, Kato H, Ueda A (2001) A miniature actuator with electromagnetic elements. Denki Gakkai Ronbunshi/Electrical Engineering in Japan 134(4): 70-75.

Tzeranis D, Papadopoulos E (2003) On the design of an autonomous robot fish. In Proceedings of 11th Mediterranean Conference on Control and Automation MED, $17-20$.

Uchino K (2006) Expansion from IT/Robotics to ecological/energy applications. In ACTUATOR06, 48. Bremen, DE.

Ueha S, Tomikawa Y (1993) Ultrasonic motors, theory and applications. Oxford Science.

Wiguna T, Heo S, Park HC, Goo NS (2006) Mechanical design of biomimetic fish robot using lipca as artificial muscle. In Key Engineering Materials, volume: Experimental Mechanics in Nano and Biotechnology, 1443-1446.

Wood RJ (2008) The first takeoff of a biologically inspired at-scale robotic insect. Transactions on Robotics 24(2): 341-347.

Yan S, Zhang F, Qin Z, Wen S (2005) A 3-DOFs mobile robot driven by a piezoelectric actuator. Smart Materials and Structures: Technical Note 15 (No. 1). 
Zesch W, Bychi R, Codourey A, Siegwart R (1995) Inertial drive for micro and nan robots: two novel mechanics. In Lynne B. Parker (ed.), Proceedings in Microrobotics and Micromechanical Systems 2593, 80-88.

Zhang L, Huang Q, Li Y, Gao J, Li H, Wu L (2012) Research and development of throw able miniature reconnaissance robot. In 2012 IEEE International Conference on Mechatronics and Automation, 1254-1259.

Zhou M, Tuo Y, Cheng L, Liu WT, Fu X (2013) A biomimetic earthworm-like micro robot using nut-type piezoelectric motor. In Sixth International Conference on Intelligent Robotics and Applications ICIRA 2013.

Zhu C (2007) In-pipe robot for inspection and sampling tasks. Industrial Robot: An International Journal 34(1): 39-45. 
